\title{
Power Efficient Beam-Forming Algorithm for Ultra-Reliable Low Latency Millimeter-Wave Communications
}

\author{
Niloofar Mehrnia* and Sinem Coleri Ergen ${ }^{\dagger}$ \\ * Koc University Ford Otosan Automotive Technologies Laboratory (KUFOTAL), Sariyer, Istanbul, Turkey, 34450 \\ Email: nmehrnia17@ku.edu.tr \\ ${ }^{\dagger}$ Department of Electrical and Electronics Engineering, Koc University, Istanbul, Turkey, 34450 \\ Email: sergen@ku.edu.tr
}

\begin{abstract}
Achieving ultra low-latency and ultra-high reliable communications at millimeter-waves (mmWaves) is challenging due to the sensitivity of this frequency band to blockage. Different space diversity schemes based on either equal gain or beam-tracking algorithms, are proposed to cope with the significant propagation losses introduced by the blockage at mmWaves. However, equal gain algorithms suffer from the low throughput as they allocate considerable gain to the nonoptimal links. On the other hand, beam-tracking algorithms suffer from the link outage as the beam-forming is redone after dropping a large amount of data. In this study we propose a new algorithm based on the prior collection of data to eliminate power consuming beam-tracking techniques while minimum diversity level ensures high received power, ultra-reliability, and low latency.
\end{abstract}

Keywords-beam-forming, diversity scheme, millimeterwaves, ultra-high reliability, ultra-low latency, .

\section{INTRODUCTION}

The fifth generation $(5 \mathrm{G})$ mobile network exploits the millimeter wave (mmWave) bands due to its enormous amount of spectrum to greatly increase communication capacity. On the other hand, $5 \mathrm{G}$ applications ranging from machine-type communications to mission-critical communications such as autonomous driving and virtual reality require ultra reliability and low latency [1]. Although, small wave length at mmWaves, allows us to place multiple antennas in a tiny area and steer the beam in the desired direction to combat significant propagation loss at mmWave [2], using directional antennas brings challenges in achieving ultra reliability due to the blockage [2],[3]. Diversity is a promising technique based on the transmission data in multiple dimensions to achieve high reliability and low latency at the cost of extra energy consumption.

Different forms of diversity techniques that can be applied to data transmission include time diversity [4], frequency diversity [5], space diversity [6], and interface diversity [7]. The gains of these diversity techniques are either adapted based on the channel properties or chosen equally. Although, in the equal gain diversity, there is no need to track the channel continuously, the method results in sub-optimal throughput due to the allocation of large transmission power to non-optimal propagation paths. On the contrary, adaptively changing the diversity gains requires real-time channel-tracking which is costly from the standpoint of power consumption.

By assuming perfect beam-tracking at upper layers, mmWave channel measurements focus on deriving statistical parameters for channel impulse response (CIR), path loss, root mean square (RMS) delay spread, and outage probability [8]-[17]. These studies either use the best direction with maximum received power [16], [17], [13], [12], [15], [18], [9] or build a diversity scheme by combining $N$ best directions with maximum powers [19], [20], [14], [21] to obtain the channel parameters. However, to have a power efficient system and consume less transmitted power, diversity level should be as minimum as possible[3], [22].

The constant beam-tracking can not achieve ultra high reliability and low latency simultaneously. The beam-tracking algorithms need to send an acknowledge packet continuously to check whether the beam is blocked or not [3], [22]. The acknowledge packet transmission rate (APTR) determines a trade-off between power and delay efficiency such that considering very high APTR, makes the system power consuming. On the other hand, with a low APTR, transmitter (Tx) and receiver $(\mathrm{Rx})$ detect that the link is lost only after dropping a large number of packets [23], and this is not tolerable for some applications with delay constraint.

The goal of this paper is to propose a novel beamforming methodology based on the prior collection of channel measurements under the blockage scenarios based on piecewise linear model (PWL) introduced in [24] to eliminate the need for continuous beam-tracking and achieve a certain level of reliability. The original contributions of this study are listed as follows:

- We collect the data for the scenarios based on PWL for different transmitter antenna rotations and different half power beam-widths (HPBWs). This data collection is performed only once before the system begins to work, to eliminate the costly continuous beam-tracking when the system is operating.

- We determine a diversity scheme containing the minimum number of best beams with the goal of achieving a certain level of reliability. For this diversity scheme, the gains are determined optimally through a linear optimization problem to attain maximum received power.

- We demonstrate the superior performance of the proposed algorithm compared to the existing equal gain and online beam-tracking diversity schemes under different scenarios via extensive simulations.

The rest of the paper is organized as follows: Section II describes the system model and assumptions considered throughout the paper. Section III presents the beam-forming methodology for choosing minimum number of best beams 
with the goal of achieving maximum average received power for a certain level of reliability. Simulation results are presented in Section IV. Finally Section V concludes the paper.

\section{SYSTEM MODEL}

The system contains transmitter-receiver pairs requiring an ultra-reliable communication at mmWaves. We assume that the position of $\mathrm{Tx}$ and $\mathrm{Rx}$ are fixed while the environment is changing over the time. To better illustrate such systems, we bring up a very common example of an office room with fixed transmitter and receiver where the human shadowing or objects in the room introduce fading to the signal or disconnect the communication for a while. Some other instances might be an intra-vehicular communication considering fixed antennas in a moving car, where the people in the car may fade the communication or the temperature increment of the engine makes the environment time-varying for the transceivers located therein. This system model can be found also in the medical cases where the transceiver nodes inside the body are placed in the fixed positions and circulation system makes the communication vulnerable to fading.

The system applies beam-forming, which essentially either equally or optimally weighs and sums signals from different directions, such that the power of received signal is maximized while never dropping under a specific threshold [25]. Space diversity is utilized to keep the received power over the threshold for achieving a high reliable communication. Over an ultra-reliable communication, the transmitted packet is guaranteed to be received at the destination with about $10-5$ to $10-9$ packet error rate, uncorrupted and in the order they were sent [26].

The system adjusts the parameters of the directional antenna, HPBW and pointing angle of Tx to Rx, to compensate the propagation loss introduced by the signal fading. Selecting the best combination of HPBW and pointing angle helps us to obtain more power at the receiver while the threshold is concerned. As the line of sight (LoS) component accounts for a sizable percentage of received power, its existence is crucial to the operation of a reliable mmWave communication [27]. However, as this link might be blocked, we need to search for some supportive links to compensate the fading effect when the LoS path is temporary or permanently blocked. Therefore other HPBWs and pointing angle pairs between $\mathrm{Tx}$ and $\mathrm{Rx}$ should be considered to find a link with the $2^{n d}, 3^{r d}$ or even higher order maximum power. Hereupon, we guarantee a high reliable communication with least probability of packet error.

The system contains a transmitter's antenna array having $N_{t}$ antenna elements. following the approach introduced in [23], it is assumed that the $l^{\text {th }}$ reflector is located in direction $\left(\phi_{t l}, \theta_{t l}\right)$ from the transmitter. As expressed in [23], the steering vector associated with direction $\left(\phi_{t l}, \theta_{t l}\right)$ is assumed $h_{l}=\left[e^{j 2 \pi f_{0} \tau_{1}\left(\phi_{t l}, \theta_{t l}\right)}, \ldots, e^{j 2 \pi f_{0} \tau_{N_{t}}\left(\phi_{t l}, \theta_{t l}\right)}\right]^{T}$, where $f_{0}$ is the carrier frequency of the signal. Also, the signal arrives from the $i^{t h}$ antenna element and $l^{t h}$ reflector with $\tau_{i}\left(\phi_{t l}, \theta_{t l}\right)$ seconds delay.Note that ${ }^{T}$ is the transpose operator. The $N_{t} \times N_{t}$ channel matrix over the $l^{t h}$ propagation path $C_{l}$ can be expressed as

$$
C_{l}=\lambda_{l} h_{l}^{T},
$$

where $\lambda$ is the channel gain over the $l^{\text {th }}$ propagation path. To estimate the channel between the transmitter and the receiver over multiple propagation paths, first we need to find the $N$ strongest paths by sweeping the predefined sectors and then refine the channel for each path [3], [22]-[23]. The channel matrix $C$ between the transmitter and the receiver over $N$ chosen propagation paths is expressed as

$$
\boldsymbol{C}=\boldsymbol{\Lambda} \boldsymbol{H}^{T},
$$

where $\boldsymbol{\Lambda}$ is $N \times N$ diagonal matrix with $\left[\operatorname{diag}\left\{\lambda_{1} \lambda_{2} \ldots \lambda_{N}\right\}\right]_{i i}=\lambda_{i}$, and $\boldsymbol{H}$ is $N_{t} \times N$ matrix defined as $\boldsymbol{H}=\left[\begin{array}{llll}h_{1} & h_{2} & \ldots & h_{N}\end{array}\right]$ [23].

\section{METHODOLOGY}

The objective of the proposed methodology is to achieve an ultra-reliable communication with minimum number of diversity level without costly real-time beam-tracking procedure. The main features of the suggested algorithm are as follows:

1) Before the system starts operation, under PWL scenarios in the environment, beam training is performed only once to find all possible links from Tx to $\mathrm{Rx}$.

2) The links with maximum powers will be weighted optimally to maximize the power over a certain threshold.

3) Since the beam-tracking is done only once to find out the best measurement setup that guarantees achieving a reliable communication, the power consuming online beam-tracking is eliminated in our algorithm.

In the proposed methodology, Algorithm 1 is executed for PWL model based on the environment in which the measurement setup is going to operate. Minimum Diversity Beam-forming (MDBA) algorithm includes two phases: in Phase-I, transmit and receive antenna arrays adjust pointing angle and HPBW to check all possible combinations to calculate the $N_{t} \times 1$ weight vector, $w_{t}$ such that the $N$ beams are steered toward the $N$ strongest propagation paths as

$$
\left[\begin{array}{llll}
h_{1} & h_{2} & \ldots & h_{N}
\end{array}\right]^{T} w_{t}=\left[\begin{array}{lllll}
\alpha 1 & \alpha 2 & \ldots & \alpha N
\end{array}\right]^{T},
$$

where $\alpha_{l}$ is a constant coefficient controlling the gain of the beam steered toward the $l^{t h}$ path. The transmit weight vector, $w_{t}$ can be expressed as

$$
w_{t}=\left(\boldsymbol{H}^{T}\right)^{-1} \boldsymbol{\alpha}
$$

where $\boldsymbol{\alpha}=\left[\begin{array}{llll}\alpha 1 & \alpha 2 \ldots \alpha N\end{array}\right]^{T}$. Consequently, the received signal, $r$, can be calculated as follows

$$
\begin{aligned}
r & =\boldsymbol{C} w_{t} s+n=\boldsymbol{\Lambda} \boldsymbol{H}^{T}\left(\boldsymbol{H}^{T}\right)^{-1} \boldsymbol{\alpha}+n \\
& =\boldsymbol{\Lambda} \boldsymbol{\alpha} s+n=\sum_{l=1}^{N} \alpha_{l} \lambda_{l} s+n,
\end{aligned}
$$

where $s$ and $n$ are the transmitted signal and additive white Gaussian noise (AWGN), respectively.

In Phase-II of MDBA, the diversity level (i.e. $N$ ) and the gains of chosen links (i.e. $\alpha_{l}$ ) are determined to satisfy the condition of reliable communication in which the received power is above a certain threshold while maximum power is utilized. The first link with maximum power is passed through the MDBA algorithm (Line 12) to check whether the power does not exceed the threshold evermore. In the case where the first link violates this condition, two links or more with maximum powers are combined according to the optimization problem presented in Eq. (6), not only to 


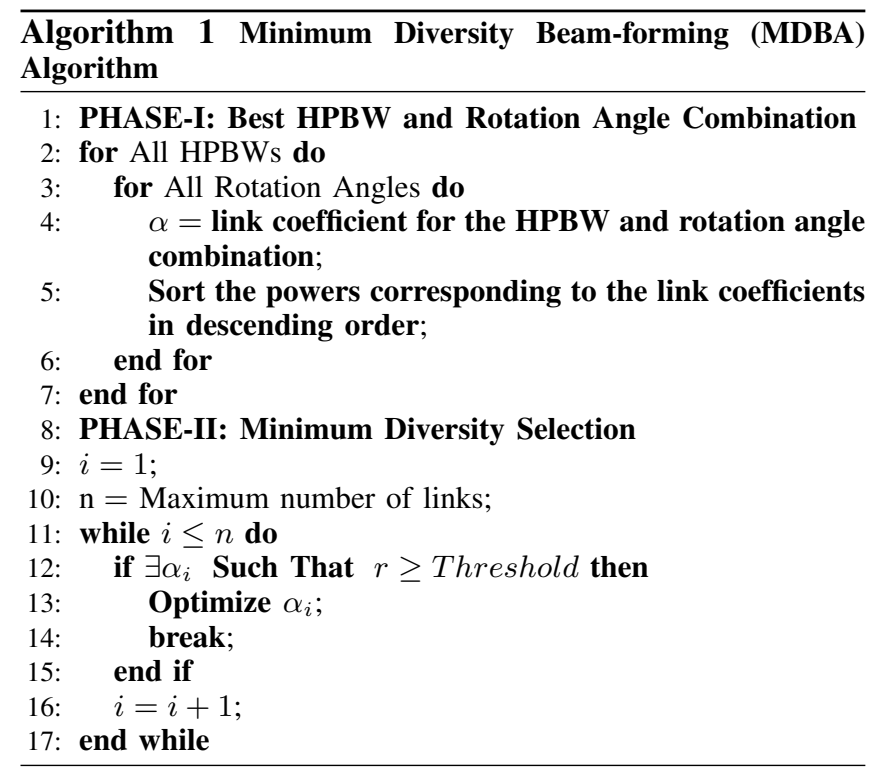

satisfy the threshold condition, but also to achieve maximum received power.

The optimal gain assignment of the best links is therefore formulated as follows:

Maximize

$$
r=\sum_{l=1}^{N} \alpha_{l} \lambda_{l} s+n
$$

subject to

$$
\sum_{i=1}^{i=N} \alpha_{i}=1
$$

variables

$$
a_{i} \geq 0, i \in Z
$$

Where $r$ is the received power of each link obtained through MDBA algorithm, $N$ is the maximum number of links, and $i$ is the index of sorted power. Also, $\alpha$ represents the unit-less link gain. The objective of the optimization problem is maximizing the received power. The sum of link gains should be one, as stated in Eq. (6b).

It is notable that there exist a dependency between rotation angle and HPBW such that if the HPBW is $\theta$, the transmitter antenna should be rotated by $\theta$ increment step. This dependency between rotation angle and HPBW is for avoiding the overlap or correlation between the links. Additionally, as a rule of thumb to find the best rotation angle, maximum power is received when the incidence angle is almost same as the reflection angle, i.e. the reflection coefficient is -1 .

\section{PERFORMANCE EVALUATION}

The goal of this section is to evaluate the performance of the proposed MDBA algorithm compared to the existing equal gain combining [23] and beam-tracking algorithms [22]. In beam-tracking algorithm, we assume that the transceiver communicates over the link with maximum received power, which is usually the LoS one. Once the link is being blocked, the communication will be switched to the link with second largest power. To make the comparison, the corresponding channel parameters are obtained by means of exploiting the channel modeling capability of a powerful electromagnetic modeling tool, named "Wireless InSite" (WI). At the first step, a 3D environment with the specific properties of objects such as wall and ceiling is created in WI. In the next step, we choose Shooting and Bouncing Ray (SBR) method which traces the rays from a transmitter and then propagates the rays through a defined environment until they reach a receiver. The rays, which are specularly reflected, have at most 30 interactions. These interactions include transmission, reflection, and diffraction around the objects [28]. Then the raw data is imported to MATLAB $^{\circledR}$ for more analysis. The summary of the simulation parameters is shown in Table I.

Table I: Summary of simulation parameters

\begin{tabular}{|c|c|}
\hline Parameter & Value \\
\hline Carrier Frequency & $60 \mathrm{GHz}$ \\
Bandwidth & $1 \mathrm{GHz}$ \\
Transmitter Power & $16 \mathrm{dBm}$ \\
Tx, Rx Height & $0.8 \mathrm{~m}$ \\
Office Dimension & $6 \mathrm{~m} \times 6 \mathrm{~m} \times 3 \mathrm{~m}$ \\
Tx Antenna Type & Directional \\
Rx Antenna Type & Omnidirectional \\
Tx Antenna Gain & 22 \\
Rx Antenna Gain & 2 \\
\hline
\end{tabular}

\section{A. Simulation Scenario}

Based on our system model, we have emulated an office room with objects in the existence of maximum 2 people. $\mathrm{Tx}$ and $\mathrm{Rx}$ are located exactly in front of each other at the same height with $4 \mathrm{~m}$ separation distance. $\mathrm{Rx}$ is on the desk, $0.8 \mathrm{~m}$ away from the ground. In the Tx position, a multiarray transmitter is placed which rotates to find the best beams with highest power level.

Fig.1 shows 3 different scenarios, which have been considered as PWL model scenarios in an office room: 1) A person walks parallel to the Tx-Rx line, 2) A person crosses the Tx-Rx line at the middle point, and 3) Two people taking different paths from door to the chairs in the office.

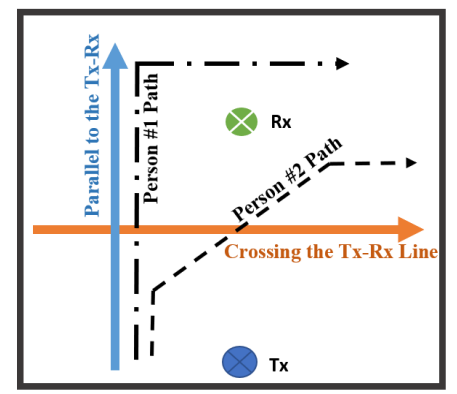

Figure 1: Different scenarios under the consideration.

To choose the proper threshold for an office environment, by referring to the IEEE $802.11 \mathrm{ad}$ standard [29] single carrier modulation and coding scheme MCS6 can deliver $1.5 \mathrm{Gbit} / \mathrm{s}$ which is enough for high definition (HD) video streaming [30]. To support this data rate or more, the received signal power has to be $-63 \mathrm{dBm}$ or higher. Therefore our requirement to have a reliable communication is to 


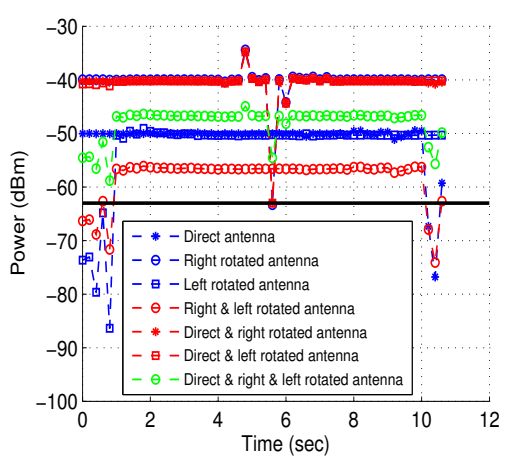

(a)

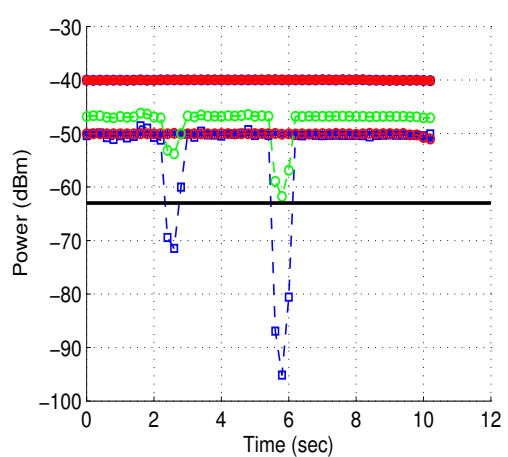

(b)

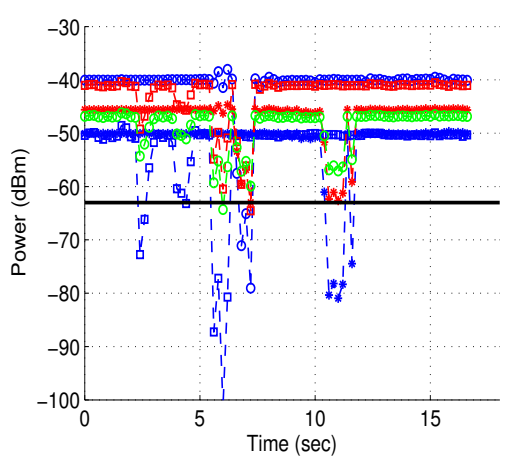

(c)

Figure 2: Received power vs. time based on equal gain combining; (a) one person crosses the direct link, (b) one person walks parallel to the direct link, and (c) two people walk in the office.

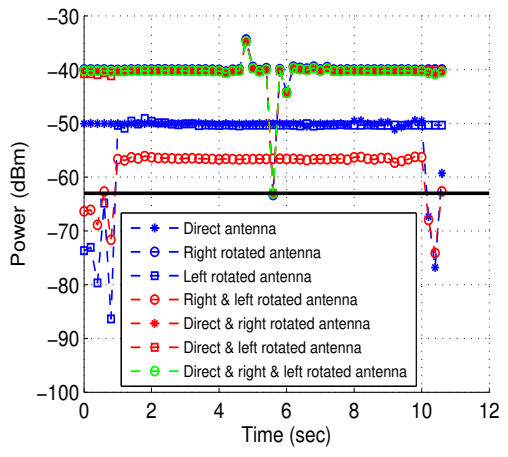

(a)

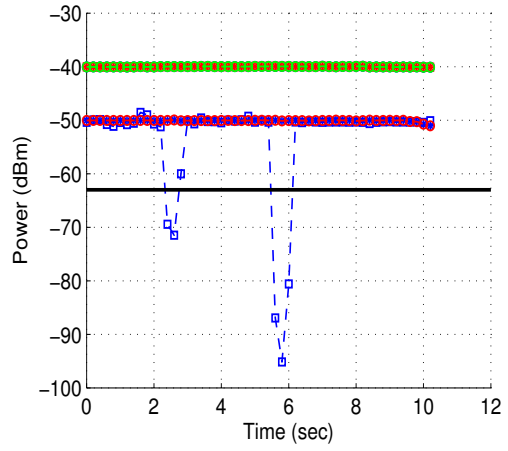

(b)

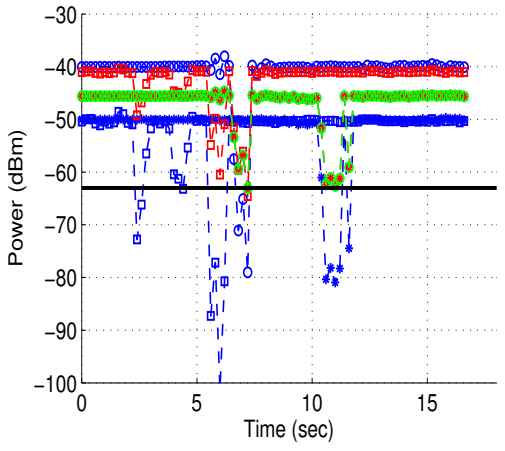

(c)

Figure 3: Received power vs. time based on MDBA Algorithm; (a) one person crosses the direct link, (b) one person walks parallel to the direct link, and (c) two people walk in the office.

receive at least $-63 \mathrm{dBm}$ power at the $\mathrm{Rx}$ point all over the time.

\section{B. Algorithm Performance}

Fig. 2 shows the received power based on equal gain algorithm for three scenarios. Only the received power obtained through the combination of 3 links, i.e. direct, right and left rotated links, is above the threshold for all scenarios. Hence, in equal gain combining, the minimum diversity level satisfying ultra-reliability is 3 . It is shown that this scheme lowers the average received power in the case of no blockage due to allocation of large transmission power to a non-optimal link.

Fig. 3 shows the received power based on MDBA algorithm for three scenarios. Among all links, the combination of direct and right rotated link guarantees ultra-reliability in all scenarios under the consideration. Moreover, the performance of this link is almost same as the combination of direct, right and left rotated links. Therefore, increasing the diversity level does not improve the communication quality significantly where optimum gains are assigned to the links in the diversity scheme. The optimum coefficients for direct and right rotated links are 0.4465 and 0.5535 , respectively. It is worth mentioning that the combination of direct and right rotated links has the best performance compared to the other, since most of the activities in the emulated office room occurred in the left side, and therefore more stable environment exists in the right side.
Fig. 4 shows the performance comparison of MDBA algorithm and the existing algorithms, i.e. equal gain and online beam-tracking [22], [23]. Fig. 4 validates the superiority of the proposed scheme over the other two algorithms. Furthermore Fig. 4 reveals that although the online beamtracking algorithm offers more throughput, the scheme suffers from the link outage, i.e. exceeding the threshold. On the other hand, not only the average throughput of MDBA algorithm is higher than that of equal gain algorithm, but also the signal level achieved by MDBA algorithm does not drop as frequently as that of equal gain algorithm. A a result, the proposed scheme does not experience any link outage, and its average received power is only $3.6 \mathrm{dBm}$ less than the averaged power obtained by online beam-tracking algorithm.

\section{CONCLUSIONS}

In this paper, we have proposed a new algorithm to eliminate the continuous beam-tracking and achieve a certain level of reliability while minimum level of diversity is utilized. We have developed a simulation platform to evaluate the performance of the proposed scheme compared to equal gain and online beam-tracking algorithms. We have shown that equal gain scheme lowers throughput in the case of no blockage, as well as online beam-tracking suffers from the link outage although it is a power consuming scheme. Extensive simulated results for different scenarios illustrate that the proposed scheme guarantees low latency ultra-reliability while maximum throughput is achieved with 


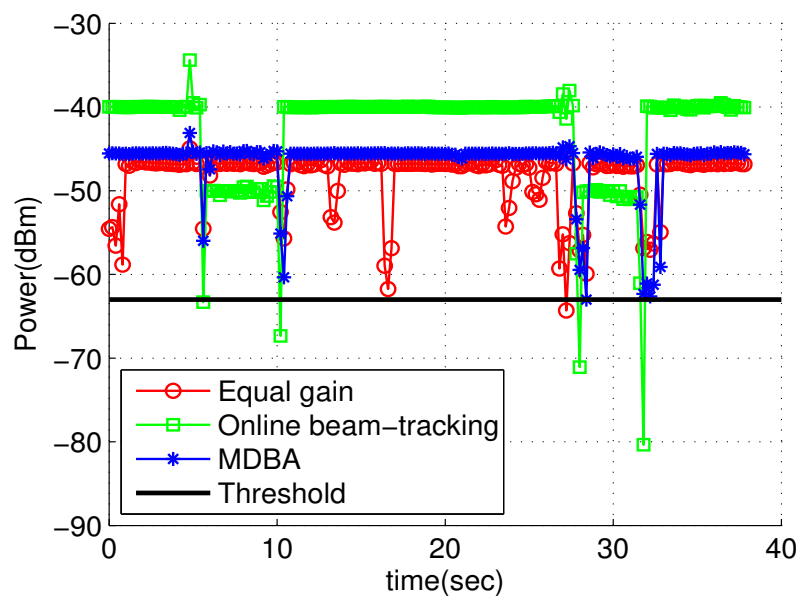

Figure 4: Performance comparison of equal gain, online beam-tracking, and MDBA algorithms.

minimum diversity level to consume less energy.

\section{ACKNOWLEDGMENT}

The authors acknowledge support from Ford Otosan. Sinem Coleri Ergen also acknowledges the financial support by METU-Prof. Dr. Mustafa Parlar Foundation Research Encouragement Award.

\section{REFERENCES}

[1] M. Bennis, M. Debbah, and H. V. Poor, "Ultrareliable and lowlatency wireless communication: Tail, risk, and scale," Proceedings of the IEEE, vol. 106, pp. 1834-1853, Oct 2018.

[2] Y. Niu, Y. Li, D. Jin, L. Su, and A. V. Vasilakos, "A survey of millimeter wave communications (mmwave) for 5G: opportunities and challenges," Wireless Networks, vol. 21, pp. 2657-2676, Nov 2015.

[3] B. Gao, Z. Xiao, C. Zhang, L. Su, D. Jin, and L. Zeng, "Doublelink beam tracking against human blockage and device mobility for 60-GHz WLAN," in 2014 IEEE Wireless Communications and Networking Conference (WCNC), pp. 323-328, April 2014.

[4] M. Serror, C. Dombrowski, K. Wehrle, and J. Gross, "Channel coding versus cooperative arq: Reducing outage probability in ultralow latency wireless communications," in 2015 IEEE Globecom Workshops (GC Wkshps), pp. 1-6, Dec 2015.

[5] G. Pocovi, B. Soret, M. Lauridsen, K. I. Pedersen, and P. Mogensen, "Signal quality outage analysis for ultra-reliable communications in cellular networks," in 2015 IEEE Globecom Workshops (GC Wkshps), pp. 1-6, Dec 2015.

[6] G. Pocovi, B. Soret, M. Lauridsen, K. I. Pedersen, and P. Mogensen, "Signal quality outage analysis for ultra-reliable communications in cellular networks," in 2015 IEEE Globecom Workshops (GC Wkshps), pp. 1-6, Dec 2015.

[7] J. J. Nielsen, R. Liu, and P. Popovski, "Ultra-reliable low latency communication using interface diversity," IEEE Transactions on Communications, vol. 66, pp. 1322-1334, March 2018.

[8] I. A. Hemadeh, K. Satyanarayana, M. El-Hajjar, and L. Hanzo, "Millimeter-wave communications: Physical channel models, design considerations, antenna constructions, and link-budget," IEEE Communications Surveys Tutorials, vol. 20, pp. 870-913, Secondquarter 2018.

[9] T. S. Rappaport, G. R. MacCartney, M. K. Samimi, and S. Sun, "Wideband millimeter-wave propagation measurements and channel models for future wireless communication system design," IEEE Transactions on Communications, vol. 63, pp. 3029-3056, Sept 2015

[10] T. S. Rappaport, Y. Xing, G. R. M. Jr., A. F. Molisch, E. Mellios, and J. Zhang, "Overview of millimeter wave communications for fifth-generation $(5 \mathrm{G})$ wireless networks-with a focus on propagation models," CoRR, vol. abs/1708.02557, 2017.
[11] S. Sun, T. S. Rappaport, T. A. Thomas, A. Ghosh, H. C. Nguyen, I. Z. Kovcs, I. Rodriguez, O. Koymen, and A. Partyka, "Investigation of prediction accuracy, sensitivity, and parameter stability of largescale propagation path loss models for $5 \mathrm{~g}$ wireless communications," IEEE Transactions on Vehicular Technology, vol. 65, pp. 2843-2860, May 2016.

[12] G. R. Maccartney, T. S. Rappaport, S. Sun, and S. Deng, "Indoor office wideband millimeter-wave propagation measurements and channel models at 28 and $73 \mathrm{ghz}$ for ultra-dense $5 \mathrm{~g}$ wireless networks," IEEE Access, vol. 3, pp. 2388-2424, 2015.

[13] G. R. MacCartney, M. K. Samimi, and T. S. Rappaport, "Exploiting directionality for millimeter-wave wireless system improvement," in 2015 IEEE International Conference on Communications (ICC), pp. 2416-2422, June 2015.

[14] T. S. Rappaport, S. Sun, R. Mayzus, H. Zhao, Y. Azar, K. Wang, G. N. Wong, J. K. Schulz, M. Samimi, and F. Gutierrez, "Millimeter wave mobile communications for $5 \mathrm{~g}$ cellular: It will work!," IEEE Access, vol. 1, pp. 335-349, 2013.

[15] S. Sun, G. R. MacCartney, M. K. Samimi, and T. S. Rappaport, "Synthesizing omnidirectional antenna patterns, received power and path loss from directional antennas for $5 \mathrm{~g}$ millimeter-wave communications," in 2015 IEEE Global Communications Conference (GLOBECOM), pp. 1-7, Dec 2015.

[16] M. K. Samimi and T. S. Rappaport, "3-d millimeter-wave statistical channel model for $5 \mathrm{~g}$ wireless system design," IEEE Transactions on Microwave Theory and Techniques, vol. 64, pp. 2207-2225, July 2016.

[17] G. R. MacCartney, T. S. Rappaport, and A. Ghosh, "Base station diversity propagation measurements at $73 \mathrm{ghz}$ millimeter-wave for $5 \mathrm{~g}$ coordinated multipoint (comp) analysis," in 2017 IEEE Globecom Workshops (GC Wkshps), pp. 1-7, Dec 2017.

[18] J. Palacios, D. D. Donno, and J. Widmer, "Tracking mm-wave channel dynamics: Fast beam training strategies under mobility," in IEEE INFOCOM 2017 - IEEE Conference on Computer Communications, pp. 1-9, May 2017.

[19] C. Slezak, A. Dhananjay, and S. Rangan, "60 ghz blockage study using phased arrays," in 2017 51st Asilomar Conference on Signals, Systems, and Computers, pp. 1655-1659, Oct 2017.

[20] M. K. Samimi and T. S. Rappaport, "Local multipath model parameters for generating $5 \mathrm{~g}$ millimeter-wave 3gpp-like channel impulse response," in 2016 10th European Conference on Antennas and Propagation (EuCAP), pp. 1-5, April 2016.

[21] S. Kwon and J. Widmer, "Multi-beam power allocation for mmwave communications under random blockage," in 2018 IEEE 87th Vehicular Technology Conference (VTC Spring), pp. 1-5, June 2018.

[22] Z. Xiao, "Suboptimal spatial diversity scheme for $60 \mathrm{GHz}$ millimeterwave WLAN," IEEE Communications Letters, vol. 17, pp. 17901793, September 2013.

[23] M. Park and H. K. Pan, "A spatial diversity technique for IEEE 802.11ad WLAN in $60 \mathrm{GHz}$ band," IEEE Communications Letters, vol. 16, pp. 1260-1262, August 2012.

[24] M. Peter, M. Wisotzki, M. Raceala-Motoc, W. Keusgen, R. Felbecker, M. Jacob, S. Priebe, and T. Krner, "Analyzing human body shadowing at $60 \mathrm{ghz}$ : Systematic wideband mimo measurements and modeling approaches," in 2012 6th European Conference on Antennas and Propagation (EUCAP), pp. 468-472, March 2012.

[25] Q. Xu, C. Jiang, Y. Han, B. Wang, and K. J. R. Liu, "Waveforming: An overview with beamforming," IEEE Communications Surveys Tutorials, vol. 20, pp. 132-149, Firstquarter 2018.

[26] J. J. Nielsen, R. Liu, and P. Popovski, "Ultra-reliable low latency communication using interface diversity," IEEE Transactions on Communications, vol. 66, pp. 1322-1334, March 2018.

[27] S. Kutty and D. Sen, "Beamforming for millimeter wave communications: An inclusive survey," IEEE Communications Surveys Tutorials, vol. 18, pp. 949-973, Secondquarter 2016.

[28] "Remcome, Wireless InSite 2.6.3." http://www.remcom.com/ wireless-insite.

[29] Wireless LAN Medium Access Control (MAC) and Physical Layer (PHY) Specifications, vol. IEEE 802.11ad, 2010.

[30] V. Semkin, M. Jacob, T. Krner, A. Bisognin, F. Ferrero, C. Luxey, and A. V. Risnen, "Estimation of optimum antenna configurations supported by realistic propagation models at $60 \mathrm{GHz}$," in The 8th European Conference on Antennas and Propagation (EuCAP 2014), pp. 3434-3438, April 2014. 